\title{
Medios de comunicación y elección presidencial 2014: una relación asistida por la coyuntura política*
}

\author{
Nathaly Montero Urrutia** \\ Recibido: 2018-10-02. Enviado a pares: 2018-10-15. \\ Aprobado por pares: 2018-11-29. Aceptado: 2018-12-15 \\ https://doi.org/10.22395/angr.v17n34a5
}

\begin{abstract}
Resumen
El presente artículo ofrece un análisis investigativo de la estructura informativa de los medios de comunicación con más audiencia en Colombia durante el proceso electoral a la presidencia 2014. Este proceso es reconocido porque la campaña electoral gravitó en torno al proceso de paz y presentó, finalmente, el abstencionismo más alto de las últimas elecciones con un $60 \%$, solo superado por las elecciones presidenciales de 1994. Este proceso se aborda desde el 15 de marzo, después de las elecciones legislativas, al 15 de junio, con el fin de tener en cuenta la primera y la segunda vuelta, así como la relación con los resultados obtenidos durante este proceso electoral. Por ende, la investigación se aborda desde el enfoque del análisis crítico del discurso (ACD) basado en los aportes del análisis del discurso en cuanto a la descripción estructural de este, pero con un interés más enfático en los problemas sociales en los que el abuso del poder es practicado, reproducido y poco refutado por los textos y el discurso político. Teniendo en cuenta que el ACD utiliza métodos empíricos y prácticos para la evaluación de los diferentes discursos, la investigación toma como referentes principales los conceptos desarrollados por teóricos como Teun Van Dijk, Gilles Gauthier y Beaudoux, D'Adamo y Slavinsky, así como los aportes hechos por la teoría de la agenda setting, puesto que asegura la influencia de los medios de comunicación sobre el público al determinar y clasificar los asuntos que poseen interés informático. Por lo tanto, se diseña una matriz con variables cuali-cuantitativas de corte comunicacional, con la finalidad de analizar si la estructura de las noticias emitidas por los medios seleccionados generó cierto clima político y suscitó que los candidatos con noticias más acordes al clima de opinión recibieran mayor cantidad de menciones y se legitimaran ante la población, frente a los candidatos que basaron su campaña en temas diferentes a los de la agenda mediática, lo que llega a vulnerar la democracia de una competencia equitativa entre candidatos, y el derecho de la audiencia para recibir información imparcial que le permita un análisis de las propuestas de todos los candidatos sin que esta haya sido previamente sesgada.

Palabras clave: medios de comunicación; candidato; proceso electoral; democracia; agenda setting; análisis crítico del discurso; audiencia; estructura informativa.
\end{abstract}

Este artículo establece una recopilación del trabajo de tesis de pregrado con mención honorífica Medios de comunicación y elección presidencial 2014: una relación asistida por la coyuntura política, con sede en la Facultad de Derecho, Ciencias Políticas y Sociales del Departamento de Ciencia Política de la Universidad del Cauca, Colombia, dirigido por el magíster Juan Carlos Varona Albán.

** Politóloga y miembro activo del Semillero Ciudad, Universidad y Juventud (CUJ), adscrito al grupo de investigación Actores, Procesos e Instituciones Políticas (Giaprip). Facultad de Derecho, Ciencias Políticas y Sociales del Departamento de Ciencia Política de la Universidad del Cauca.nmontero@unicauca.edu.co. Orcid: https://orcid.org/00000002-3638-1701 


\title{
Media and the 2014 presidential election: a relationship aided by the political situation
}

\begin{abstract}
This article presents a research on the information structure of the media with the highest audience rating in Colombia during the 2014 presidential election in Colombia. This process is widely recognized because the election campaign revolved around the peace process and had the highest abstentionism in recent years (60\%), only surpassed by the 1994 presidential election. The process is analyzed from March 15 (after the legislative elections) to June 15, in order to consider both the first and second rounds, as well as the relationship with the results obtained during this electoral process. Therefore, the research was carried out with the critical discourse analysis (CDA) approach, based on the contributions of discourse analysis in terms of its structural description, but with a more stressed interest in social problems in which the abuse of power is practiced, reproduced and scarcely refuted by texts and political discourse. Considering that CDA uses empirical and practical methods for evaluating different discourses, the research takes the concepts developed by theoreticians such as Teun Van Dijk, Gilles Gauthier, Beaudoux, D'Adamo and Slavinsky, as well as the contributions of the agenda setting theory, as key referents, because that ensures the influence of the media on the public in determining and classifying the issues that have media interest. Therefore, a matrix with qualitativequantitative variables of communicational nature was designed, aimed at analyzing whether the news' structure issued by the selected media spawned a particular political climate, and caused that candidates whose news were more in line with the climate of opinion receive more mentions and be legitimized before the population, compared to the candidates who campaigned based on issues not related to the media agenda, which ends up undermining the democracy of a fair competition between candidates, and the right of the audience to receive impartial information that allows an analysis of the proposals of all candidates without it having been previously distorted.
\end{abstract}

Keywords: media; candidate; electoral process; democracy; agenda setting; critical discourse analysis; audience; informative structure.

\section{Meios de comunicação e eleição presidencial 2014: uma relação assistida pela conjuntura política}

\begin{abstract}
Resumo
O presente artigo oferece uma análise investigativa da estrutura informativa dos meios de comunicação com mais audiência na Colômbia durante o processo eleitoral à presidência 2014. Esse processo é reconhecido porque a campanha eleitoral gravitou em torno ao processo de paz e apresentou, finalmente, o abstencionismo mais alto das últimas eleições (60\%), superado apenas pelas eleições presidenciais de 1994. Esse processo é abordado desde 15 de março, depois das eleições legislativas, até 15 de junho, com o fim de levar em consideração o primeiro e o segundo turno, bem como a relação com os resultados obtidos durante esse processo eleitoral. Portanto, a pesquisa é realizada a partir da abordagem da análise crítica do discurso (ACD) com base nas colaborações da análise do discurso quanto à sua descrição estrutural, mas com um interesse mais enfático nos problemas sociais nos quais o abuso de poder é praticado, reproduzido e pouco refutado pelos textos e pelo discurso político.

Ao levar em consideração que a ACD utiliza métodos empíricos e práticos para a avaliação dos diferentes discursos, a pesquisa toma como principais referentes os conceitos desenvolvidos por teóricos como Teun Van Dijk, Gilles Gauthier e Beaudoux, D'Adamo e Slavinsky, bem como as colaborações feitas pela teoria da agenda setting, posto que garante a influência dos meios de comunicação sobre o público ao determinar e classificar os assuntos de interesse informático. Portanto, foi criada uma matriz com variáveis quali-quantitativas de corte comunicacional, com a finalidade de analisar se a estrutura das notícias emitidas pelos meios selecionados gerou certo clima político e suscitou que os candidatos com notícias mais adequadas ao clima de opinião recebessem maior quantidade de menções e se legitimassem ante a população em comparação com os candidatos que basearam sua campanha em temas diferentes aos da agenda midiática. Isso chega a vulnerar a democracia de uma competência igualitária entre candidatos e o direito da audiência de receber informação imparcial que lhe permita uma análise das propostas de todos os candidatos sem que esta tenha sido previamente distorcida.
\end{abstract}

Palavras-chave: meios de comunicação; candidato; processo eleitoral; democracia; agenda setting; análise crítica do discurso; audiência; estrutura informativa. 


\section{Introducción}

Las elecciones en Colombia, a gran escala, se reflejan como una democracia electoral, puesto que los gobernantes de turno, a través de los años, han sido elegidos mayoritariamente por la población que participa de forma activa el día de la elección. Sin embargo, Colombia presenta varios problemas, que si bien es cierto no son exclusivos de este país, han estado presentes como ejes a solucionar en las campañas de los diferentes periodos de gobierno. El primero, hace referencia a los grupos guerrilleros, paramilitares y bandas criminales que han generado violencia extrema con sus actos en el país. El segundo, se refiere al tráfico de drogas a nivel internacional que tiene la producción y manipulación originalmente desde el país. Por último, la corrupción en las tres ramas de poder político que ha ocasionado deslegitimación institucional, así como desigualdad social y económica. "Las dinámicas electorales colombianas de las últimas décadas, han tenidos presencia de constante violencia política que, junto con el conflicto armado, ponen a prueba cada cuatro años la solidez de la democracia formal del país y de sus instituciones representativas" (Tamayo, 2007, p. 119).

Juan Manuel Santos, presidente electo desde el 2010, y quien buscaba la reelección en el 2014, se propuso iniciar conversaciones de paz con las Fuerzas Armadas Revolucionarias de Colombia (FARC) que son, según Serrano (2014), uno de los grupos guerrilleros con más trayectoria y con un alto número de militantes (superior a los 15 mil hombres). De esta manera, Santos convirtió el proceso de paz en su ficha clave para ganar la reelección teniendo en cuenta que su imagen era desfavorable en un 58 \% dentro del proceso electoral (Semana, 2014).

El proceso electoral a presidencia en Colombia 2014 empieza a tener relevancia mediática cuando el Centro Democrático, partido opositor de la campaña reeleccionista, logra un alto número de curules en el Senado, lo que permitió que Óscar Iván Zuluaga, como candidato del Centro Democrático, ganara fuerza electoral y se convirtiera en el principal contrincante del candidato presidente y de la forma como se estaban desarrollando los diálogos de paz. Y es justo en este momento cuando el contexto que plantearon los medios giró en torno a los problemas que se presentaron entre las campañas de Zuluaga y Santos, y relegaron temas importantes como el desempleo, la salud, la vivienda, la educación, entre otros. Los candidatos Clara López y Enrique Peñalosa respaldaron el proceso de paz, pero buscaron enfocar su campaña en temas alternos a los de la agenda setting; Marta Lucía Ramírez tuvo una posición más crítica frente al proceso de paz, sin embargo, no toma como línea estratégica este asunto y basa su campaña política en temas generales que estuvieron en contra y a favor del gobierno Santos, pero que no eran primordiales en la agenda de los medios seleccionados.

Bajo este panorama, el balotaje se dio entre las dos campañas más sonadas en primera vuelta, es decir, la de Santos y la de Zuluaga, los medios tenían en su agenda, como tema principal, el proceso de paz y se promovió una polarización del país que ya se venía gestando desde las denuncias entre las dos campañas; los resultados favorecían a Santos 
como el presidente electo para 2014-2018. Es pertinente aclarar que desde autores como Lazarsfeld, Berelson y Gaudet (1949) con su investigación The peoples choice. How the Voter Makes up his Mind in a Presidential Campaign se plantean otros factores de vital importancia en la elección de un presidente como lo es el grado de escolaridad, la clase social y económica, la ideología, la cultura, los sentimientos políticos, entre otros. Sin embargo, el enfoque de la investigación es analizar ¿Cómo fue la estructura informativa de los medios de comunicación seleccionados y su relación con los resultados obtenidos en el proceso electoral para presidencia en Colombia, 2014?

Es primordial entender que los medios de comunicación sirven como canales trasmisores de información que pretenden lograr un efecto en el receptor, "mucho más que medios de distribución, los medios de comunicación son espacios de producción de los discursos que configuran la realidad social" (González, 1989, p. 13) lo cual generó el interés por el estudio hacia los medios de comunicación y la elaboración de diferentes teorías sobre su poder en la población. Entre las teorías más importantes se destaca "la aguja hipodérmica" de Harold Laswell, para 1920 aseguraba que los medios tenían la capacidad de moldear conductas y de estimular a las masas que no poseían algún criterio.

A principios de los años 70 nace la teoría de la agenda setting por los autores McCombs y Shaw (1977) que reconoce cierto poder de los medios de comunicación, ya que se utilizan categorías que generan un estatus de los actores políticos dentro de la población, se oculta información y se expone otra de manera planificada, todo esto termina generando un determinado clima político. Los autores aseguran que una campaña política es un lugar clave para estudiar la influencia de los medios masivos porque el comportamiento está conectado con cómo nuestro entorno político es percibido (Aceves, 1993).

Los medios constituyen a la vez un elemento estructural y estructurante del espacio público (...), en tanto que no solo actúan dentro de este espacio sino que pueden modificarlo mediante sus acciones, operan con libertad respaldados en saber que su labor es sinónimo de poder, porque si bien solo recrean la actividad política (...) generan un discurso cuyo resultado puede ser bien, de ayudante del político y partido en cuestión, bien, de opositor (...) e incidir de esta forma en la cultura y participación política de los ciudadanos (Verón y Velázquez, 1992 citados por Vega, 2010, p.17).

Los noticieros tienen una superestructura esquemática que consiste en un cierto número de categorías que permiten establecer cierto orden en la jerarquización de noticias, así, las más importantes o interesantes tienen mayor probabilidad de aparecer en primera plana, o como noticia relevante en todos los medios masivos, ocasionando, tal como es descrito por Gauthier (1998), que se genere una relación causal entre la importancia que los medios le dan a cierta información y la percepción que adquieren los consumidores de la importancia de estas cuestiones, lo que para muchos autores, se convierte en cierta influencia para los espectadores en cuanto a la opinión respecto a los sucesos que se estén presentando en una coyuntura tanto nacional como internacional. Problema que también es vivido en los procesos de campaña electoral, puesto que tal como es planteado por 
Van Dijk (1980) "la selección de actores importantes en las noticias depende de su poder político y social, de su accesibilidad o de muchas otras condiciones sociopolíticas" (p. 177). Esto produce que, para los procesos electorales, los candidatos deban tener una fuerte maquinaria política y lograr venderse mediáticamente para poder llegar a ocupar los primeros lugares en prensa, radio y televisión.

El presente artículo está basado en un análisis sobre el contenido noticioso de los medios seleccionados ${ }^{1}$, es decir Caracol Televisión, Caracol Radio y el periódico El Tiempo, los cuales presentaron los más altos niveles de audiencia durante el periodo tiempo electoral estudiado. Bajo el enfoque del ACD y la teoría de la agenda setting, se analizan los contenidos noticiosos de cada uno de los medios a través de una base de datos cualitativa con variables de estudio tomadas desde los aportes de diferentes autores, para posteriormente cuantificar los datos obtenidos con el fin de encontrar algunos patrones comunes entre la estructura informativa de los medios y el resultado obtenido por los candidatos presidenciales.

\section{Metodología}

Para analizar la estructura informativa de los medios de comunicación seleccionados, se parte de una subdivisión del tiempo de estudio elegido, es decir, para los tres medios se analizan las noticias desde el 15 de marzo al 15 de junio, las cuales se subdividen en cuatro periodos de tiempo que van del 15 de marzo al 14 de abril, del 15 de abril al 14 de mayo, del 15 de mayo al 25 de mayo cuando finaliza la primera vuelta. Y del 26 de mayo al 15 de junio cuando finaliza la segunda vuelta. Esto con el fin de lograr variaciones más visibles entre las variables de estudio y los respectivos candidatos.

Caracol Televisión presentó un total de 103 entradas entre el 15 de marzo al 25 de mayo que finaliza la primera vuelta, y 34 entradas entre el 26 de mayo al 15 de junio correspondientes a la segunda vuelta. Por su parte, el periódico El Tiempo tuvo un total de 77 entradas entre el 15 de marzo al 25 de mayo, y 38 entradas entre el 26 de mayo al 15 de junio para segunda vuelta. Por último, Caracol Radio contó con 39 entradas entre el 15 de marzo al 25 de mayo, y con 27 entradas en segunda vuelta.

Las entradas de cada medio se organizaron en una base de datos cualitativa para cada uno, es decir, una base de datos para Caracol Televisión, una base de datos con El Tiempo y otra para Caracol Radio. Las bases de datos cuentan con casillas de información sobre la noticia como: día, mes, año, título y contenido. Igualmente, los tres medios trabajan con las mismas categorías y variables de análisis con el fin de cuantificar los resultados obtenidos por cada medio y hacer un ejercicio comparativo entre la estructura informa-

Los datos de televisión fueron obtenidos de la página web: www.ratingcolombia.com, para radio se tomó en cuenta un reporte de la revista Semana sobre la encuesta ECAR, para más información consultar: http://www.semana.com/ nacion/articulo/emisoras-con-mayor-audiencia-en-colombia-segun-ecar/381022-3. Por último, los resultados en prensa se tomaron del Estudio General de Medios hecho en el 2014 del cual el periódico El Tiempo saca reporte, para más información consultar: http://www.eltiempo.com/archivo/documento/CMS-14031815 
tiva de los tres medios, así como una comparación entre las noticias emitidas para cada candidato por medio.

Las categorías y variables utilizadas para el análisis de la estructura informativa de los medios son tomadas desde referentes teóricos como: García, V., D’Adamo, O. y Slavinsky, J. (2005) con su libro Comunicación política y campañas electorales, de igual manera, se propuso algunas variables que fueron de elaboración propia bajo teorías como las de Gilles Gauthier con Comunicación y política y Teun Van Dijk con La noticia como discurso, comprensión, estructura y producción de la información y Estructura y funciones del discurso, con el fin de comprobar si la estructura informativa de las noticias emitidas durante el periodo de tiempo elegido por los medios seleccionados, generó estatus entre los candidatos y cierto clima político que suscitó un determinado resultado.

Tabla 1. Variables tomadas en cuenta dentro de esta investigación

\begin{tabular}{cc}
\hline Variable & Categoríalítem \\
\hline
\end{tabular}

Menciones dato es nombrado por los medios de comunicación en sus noticias durante el periodo de tiempo analizado.

Lenguaje

(Beaudoux, D’Adamo Presente, pasado y Slavinsky)

Hace referencia a anuncios positivos que abordan temas del futuro o del presente

Hace referencia a anuncios que en su mayoría son negativos con un más difícil de captar

Rara vez mencionan a los contrincantes y promueven la cara favorable del candidato

Es un anuncio positivo que marca diferencias con los demás candidatos, existe el de contraste directo:

Tipo de anuncio (Beaudoux, D’Adamo Contraste y Slavinsky) cuando comparan la posición de un candidato con el otro, y de contraste implícito: no hacen referencia explícita a la oposición sino que se apuesta a la deducción del elector

Anuncios de ataque directo a la oposición fijando Negativo el foco en debilidades personales o de gestión del adversario

Coyuntura política (CP) Hace referencia a la opinión de los candidatos frente al proceso de paz y sobre su campaña (primera vuelta), proceso de paz (segunda vuelta)

Hace referencia a la posición o propuestas del candiTema Cotidianidad (C) dato frente asuntos políticos, económicos, sociales y laborales que vive el país

Caracterización so- Hace referencia a las noticias donde se expone el cial y física del can - ámbito familiar y social del candidato didato (CSFC) 


\begin{tabular}{|c|c|c|}
\hline Variable & Categoría/ítem & Significado \\
\hline \multirow{8}{*}{$\begin{array}{l}\text { A quién va dirigido el } \\
\text { anuncio } \\
\text { (Beaudoux, D'Adamo } \\
\text { y Slavinsky). }\end{array}$} & $\begin{array}{l}\text { Dirigentes políticos } \\
\text { (DP) }\end{array}$ & \\
\hline & Público general (PG) & $\begin{array}{l}\text { Hace referencia a las noticias en donde no se especi- } \\
\text { fica un grupo de personas }\end{array}$ \\
\hline & $\begin{array}{l}\text { Estudiantes } \\
\text { universitarios (EU) }\end{array}$ & \\
\hline & $\begin{array}{l}\text { Fuerzas de } \\
\text { seguridad (FS) }\end{array}$ & \\
\hline & Mujeres (M) & \\
\hline & Profesionales (P) & \\
\hline & $\begin{array}{l}\text { Campesinos, } \\
\text { indígenas y afro } \\
\text { (CIA) }\end{array}$ & $\begin{array}{l}\text { De acuerdo al contexto social colombiano, se hace } \\
\text { necesario implementar un grupo de esta especificidad }\end{array}$ \\
\hline & $\begin{array}{l}\text { Víctimas de } \\
\text { violencia (VV) }\end{array}$ & $\begin{array}{l}\text { De acuerdo al contexto político colombiano, se hace } \\
\text { necesario implementar un grupo de esta especificidad }\end{array}$ \\
\hline
\end{tabular}

Fuente: elaboración propia

\section{Resultados de la primera vuelta}

De esta manera, el análisis se hizo por variables para los tres medios tanto en primera como segunda vuelta, lo cual le permite al lector una facilidad en la lectura y comprensión del análisis de resultados para los candidatos en cada uno de los tres medios tenidos en cuenta. Es pertinente resaltar que los resultados expuestos para cada uno de los candidatos en los tres medios, son el promedio obtenido de la sumatoria de sus porcentajes en cada uno de los subperiodos nombrados anteriormente. Igualmente, cada candidato está representado en las tablas por el color de su partido junto a la inicial de su apellido, así: Juan Manuel Santos (S), color naranja; Óscar Iván Zuluaga (Z), color azul claro; Marta Lucía Ramírez (R), color azul oscuro; Clara López (L), color amarillo; Enrique Peñalosa (P), color verde.

En la tabla 2 se muestra el resultado en menciones que tuvo cada uno de los candidatos por cada medio para primera vuelta, las menciones permiten saber qué tan frecuente es un candidato expuesto mediáticamente a través de las noticias y según Neumann (1995) los candidatos que logran obtener la mayor atención de los medios, se legitiman ante la población y por esta misma razón tienen mayores posibilidades de convertirse en los más celebres y lograr su objetivo principal

Caracol Televisión, durante los tres periodos de tiempo seleccionados, tuvo 103 noticias en donde se mencionó 255 veces a los candidatos, Zuluaga fue el candidato con más menciones y Ramírez la candidata con menos, sin embargo, la diferencia porcentual entre los candidatos no es tan visible como en los otros medios masivos tomados. El periódico 
El Tiempo tuvo un resultado de 77 noticias respecto al proceso electoral, se mencionó 171 veces a los candidatos. En este caso fue Santos el candidato con más menciones, mientras que López fue la candidata menos mencionada durante los tres periodos con una diferencia porcentual entre los candidatos más amplia que la de Caracol Televisión, de 14,3 \%. Por parte de Caracol Radio se tuvieron un total de 39 noticias en las cuales se nombró 58 veces a los candidatos, los más nombrados fueron Zuluaga y Santos mientras que López vuelve a ser la candidata con menos menciones y con una diferencia porcentual respecto a Zuluaga de 29,6 \% y Santos de $21,3 \%$.

Tabla 2. Total de menciones para cada candidato por medio en primera vuelta

\begin{tabular}{|c|c|c|}
\hline \multicolumn{2}{|c|}{ Candidato X medio $\quad$ Variable } & Menciones \\
\hline \multirow{5}{*}{ Caracol Televisión } & Juan Manuel Santos (S) & $19,6 \%$ \\
\hline & Óscar Iván Zuluaga (Z) & $23,5 \%$ \\
\hline & Marta Lucía Ramírez (R) & $18,1 \%$ \\
\hline & Clara López (L) & $18,8 \%$ \\
\hline & Enrique Peñalosa (P) & $18,6 \%$ \\
\hline \multirow{5}{*}{ El Tiempo } & Juan Manuel Santos (S) & $28,7 \%$ \\
\hline & Óscar Iván Zuluaga (Z) & $23,6 \%$ \\
\hline & Marta Lucía Ramírez (R) & $15,9 \%$ \\
\hline & Clara López (L) & $14,4 \%$ \\
\hline & Enrique Peñalosa (P) & $16,5 \%$ \\
\hline \multirow{5}{*}{ Caracol Radio } & Juan Manuel Santos (S) & $30 \%$ \\
\hline & Óscar Iván Zuluaga (Z) & $38,3 \%$ \\
\hline & Marta Lucía Ramírez (R) & $10,4 \%$ \\
\hline & Clara López (L) & $8,7 \%$ \\
\hline & Enrique Peñalosa (P) & $12,4 \%$ \\
\hline
\end{tabular}

Fuente: elaboración propia

Teniendo en cuenta lo anterior, las agendas de los medios analizados favorecieron a los candidatos Santos y Zuluaga, los cuales, en medios como El Tiempo y Caracol Radio, no ocuparon los últimos lugares en menciones. Mientras que Ramírez, López y Peñalosa, con considerables diferencias porcentuales respecto a Santos y Zuluaga, se disputaron los últimos lugares en menciones, esto permite entender que "los políticos para ser reconocidos ya no necesitan poseer convicción, sino acumular apariciones" (Berrocal, 2008, p. 8). En otras palabras, los candidatos que logran obtener la mayor atención de los medios, son los que según Neumann (1995) se legitiman ante la población, y por esta misma razón tienen mayores posibilidades de convertirse en los más celebres y lograr su objetivo principal. 
Tabla 3. Resultado de candidato por medio en primera vuelta con las variables lenguaje y tipo de anuncio

\begin{tabular}{|c|c|c|c|c|c|c|}
\hline \multirow[b]{2}{*}{ Candidato X medio } & \multirow[t]{2}{*}{ Variable } & \multicolumn{2}{|c|}{ Lenguaje } & \multicolumn{3}{|c|}{ Tipo de anuncio } \\
\hline & & Presente & Pasado & Positivo & Contraste & Negativo \\
\hline \multirow{5}{*}{$\begin{array}{l}\text { Caracol } \\
\text { Televisión }\end{array}$} & Juan Manuel Santos (S) & $19,6 \%$ & $0 \%$ & $8,6 \%$ & $4 \%$ & $4,2 \%$ \\
\hline & Óscar Iván Zuluaga (Z) & $21,2 \%$ & $2,3 \%$ & $12,6 \%$ & $5,5 \%$ & $5,1 \%$ \\
\hline & Marta Lucía Ramírez (R) & $17,1 \%$ & $1 \%$ & $10,7 \%$ & $5 \%$ & $1,4 \%$ \\
\hline & Clara López (L) & $17,9 \%$ & $1 \%$ & $14,1 \%$ & $2,9 \%$ & $1 \%$ \\
\hline & Enrique Peñalosa (P) & $17,6 \%$ & $1 \%$ & $113 \%$ & $2,6 \%$ & $2,6 \%$ \\
\hline \multirow{5}{*}{ El Tiempo } & Juan Manuel Santos (S) & $27,7 \%$ & $1 \%$ & $11,7 \%$ & $4,1 \%$ & $9,3 \%$ \\
\hline & Óscar Iván Zuluaga (Z) & $21,9 \%$ & $1,7 \%$ & $8,8 \%$ & $2,8 \%$ & $9,8 \%$ \\
\hline & Marta Lucía Ramírez (R) & $14,9 \%$ & $1 \%$ & $6,3 \%$ & $3,6 \%$ & $3,3 \%$ \\
\hline & Clara López (L) & $13 \%$ & $1,4 \%$ & $8,8 \%$ & $0 \%$ & $2,6 \%$ \\
\hline & Enrique Peñalosa (P) & $15,5 \%$ & $1 \%$ & $6,6 \%$ & $1,6 \%$ & $4,5 \%$ \\
\hline \multirow{5}{*}{ Caracol Radio } & Juan Manuel Santos (S) & $30 \%$ & $0 \%$ & $6,5 \%$ & $3,4 \%$ & $13,1 \%$ \\
\hline & Óscar Iván Zuluaga (Z) & $38,3 \%$ & $0 \%$ & $5 \%$ & $6,7 \%$ & $16,1 \%$ \\
\hline & Marta Lucía Ramírez (R) & $10,4 \%$ & $0 \%$ & $1,5 \%$ & $1,7 \%$ & $1,9 \%$ \\
\hline & Clara López (L) & $8,7 \%$ & $0 \%$ & $5,4 \%$ & $0 \%$ & $0 \%$ \\
\hline & Enrique Peñalosa (P) & $12,4 \%$ & $0 \%$ & $0 \%$ & $3,2 \%$ & $1,9 \%$ \\
\hline
\end{tabular}

Fuente: elaboración propia

Caracol Televisión, durante los tres periodos de tiempo seleccionados, tuvo 103 noticias en las que se mencionó 255 veces a los candidatos. Zuluaga fue el candidato con más menciones y Ramírez la candidata con menos, sin embargo, la diferencia porcentual entre los candidatos no es tan visible como en los otros medios masivos tomados. El periódico El Tiempo tuvo un resultado de 77 noticias respecto al proceso electoral, se mencionó 171 veces a los candidatos. En este caso fue Santos el candidato con más menciones, mientras que López fue la candidata menos mencionada durante los tres periodos con una diferencia porcentual entre los candidatos más amplia que la de Caracol Televisión, de 14,3\%. Por parte de Caracol Radio se tuvieron un total de 39 noticias en las cuales se nombró 58 veces a los candidatos, los más nombrados fueron Zuluaga y Santos mientras que López vuelve a ser la candidata con menos menciones y con una diferencia porcentual respecto a Zuluaga de $29,6 \%$ y Santos de $21,3 \%$.

Teniendo en cuenta lo anterior, las agendas de los medios analizados favorecieron a los candidatos Santos y Zuluaga, los cuales, en medios como El Tiempo y Caracol Radio, ocuparon los primeros lugares en menciones. Mientras que Ramírez, López y Peñalosa, con considerables diferencias porcentuales respecto a Santos y Zuluaga, se disputaron los últimos lugares en menciones, esto permite entender que "los políticos para ser reconocidos ya no necesitan poseer convicción, sino acumular apariciones" (Berrocal, 
2008, p. 8). En otras palabras, los candidatos que logran obtener la mayor atención de los medios, son los que según Neumann (1995) se legitiman ante la población, y por esta misma razón tienen mayores posibilidades de convertirse en los más célebres y lograr su objetivo principal.

En los tres medios se evidencia que los porcentajes más altos para todos los candidatos respecto a la variable de lenguaje, son noticias con lenguaje en presente, es decir, noticias que tienden a ser positivas y simples que son más fáciles de captar por el receptor. No obstante, Zuluaga es quien tuvo, respecto a sus homólogos, más noticias con lenguaje en pasado, las cuales hacen referencia a noticias que tienden a ser negativas para el candidato con una construcción de lenguaje complejo para el receptor.

Santos y Zuluaga se destacaron por ser los candidatos con más altos porcentajes de anuncios negativos y de contraste en los tres medios durante los tres periodos de tiempo, ya que estos anuncios negativos son entendidos como noticias de ataque directo hacia un mismo candidato o de un candidato hacia su homólogo. Se puede deducir que la importancia obtenida por estos dos candidatos se basó en los ataques permanentes por parte de las dos campañas durante los dos periodos de tiempo finales para culminar la primera vuelta, tal como es evidente en la tabla 4.

Tabla 4. Resultado de candidato por medio en primera vuelta con la variable tema

\begin{tabular}{|c|c|c|c|c|}
\hline \multirow{2}{*}{ Candidato X medio } & & \multicolumn{3}{|c|}{ Tema } \\
\hline & & $\mathrm{CP}$ & $\mathrm{C}$ & CSFC \\
\hline \multirow{5}{*}{ Caracol Televisión } & Juan Manuel Santos (S) & $15,6 \%$ & $3,1 \%$ & $0,4 \%$ \\
\hline & Óscar Iván Zuluaga (Z) & $15,5 \%$ & $7,3 \%$ & $0,4 \%$ \\
\hline & Marta Lucía Ramírez (R) & $9,20 \%$ & $8,9 \%$ & $0 \%$ \\
\hline & Clara López (L) & $9,20 \%$ & $9,6 \%$ & $0 \%$ \\
\hline & Enrique Peñalosa (P) & $11,1 \%$ & $7,5 \%$ & $0 \%$ \\
\hline \multirow{5}{*}{ El Tiempo } & Juan Manuel Santos (S) & $26,5 \%$ & $2,2 \%$ & $0 \%$ \\
\hline & Óscar Iván Zuluaga (Z) & $18 \%$ & $4,8 \%$ & $0,7 \%$ \\
\hline & Marta Lucía Ramírez (R) & $11,8 \%$ & $3,3 \%$ & $0,7 \%$ \\
\hline & Clara López (L) & $8,6 \%$ & $5,9 \%$ & $0 \%$ \\
\hline & Enrique Peñalosa (P) & $13,8 \%$ & $2,1 \%$ & $0,5 \%$ \\
\hline \multirow{5}{*}{ Caracol Radio } & Juan Manuel Santos (S) & $30 \%$ & $0 \%$ & $0 \%$ \\
\hline & Óscar Iván Zuluaga (Z) & $36,4 \%$ & $1,9 \%$ & $0 \%$ \\
\hline & Marta Lucía Ramírez (R) & $8,9 \%$ & $1,5 \%$ & $0 \%$ \\
\hline & Clara López (L) & $7,2 \%$ & $1,5 \%$ & $0 \%$ \\
\hline & Enrique Peñalosa (P) & $12,4 \%$ & $0 \%$ & $0 \%$ \\
\hline
\end{tabular}

Fuente: elaboración propia 
La mayoría de las noticias para los tres medios se basaron en temas de coyuntura política, es decir, se informó sobre el desarrollo de las campañas para la primera vuelta y la paz. Aunque en menor proporción, las noticias sobre cotidianidad y personificación del actor, también se hicieron evidentes y con mucha más frecuencia en los candidatos López, Ramírez y Peñalosa. Esto indica, según Rodríguez (2011), que los mensajes que estuvieron acorde al clima de opinión fueron más eficaces que los que se hicieron acorde a un punto de vista minoritario, esto evidencia cómo la agenda mediática impuso un tema frente a los temas de interés cotidiano. Tomando esto desde Lyengar y Kinder (1987), se dice que los medios establecen en la agenda temas en los que la población pueda juzgar a los personajes expuestos, y tal como expone Gauthier (1998), cuando ciertos temas sobresalen entre otros en las campañas electorales, los medios impulsan al votante a juzgar a los candidatos con relación a los temas expuestos, lo cual provoca que los candidatos que no estén dentro de la coyuntura mediática pierdan importancia y legitimidad ante la población.

La jerarquización temática de los medios en la que las campañas de desprestigio ocuparon un lugar importante, terminó por convertirse en un tema de opinión pública, haciendo que los candidatos implicados se dedicaran solo a atacar y responder los ataques de su homólogo, lo cual demostró que la agenda política al mando de un candidato presidente, se superpuso a la mediática y esta a su vez, empezó a hacer parte de la opinión pública.

Tabla 5. Resultado de candidato por medio en primera vuelta con la variable: a quién va dirigido

\begin{tabular}{|c|c|c|c|c|c|c|c|c|c|}
\hline \multirow{2}{*}{\multicolumn{2}{|c|}{ Candidato X medio $\quad$ Variable }} & \multicolumn{8}{|c|}{ A quién va dirigido } \\
\hline & & \multirow{2}{*}{ DP } & \multirow{2}{*}{$\frac{P G}{13,5 \%}$} & \multirow{2}{*}{$\frac{\mathrm{EU}}{0,4 \%}$} & \multirow{2}{*}{$\begin{array}{c}\text { FS } \\
0,3 \%\end{array}$} & \multirow{2}{*}{$\frac{M}{0,3 \%}$} & \multirow{2}{*}{$\begin{array}{c}\text { CIA } \\
0,5 \%\end{array}$} & \multirow{2}{*}{$\frac{\mathrm{VV}}{0,4 \%}$} & \multirow{2}{*}{$\begin{array}{c}\mathrm{PF} \\
0,3 \%\end{array}$} \\
\hline \multirow{5}{*}{$\begin{array}{l}\text { Caracol } \\
\text { TV }\end{array}$} & Juan Manuel Santos (S) & & & & & & & & \\
\hline & Óscar Iván Zuluaga (Z) & $6,9 \%$ & $13,5 \%$ & $1,2 \%$ & $0,7 \%$ & $0,3 \%$ & $1,1 \%$ & $1,3 \%$ & $0,4 \%$ \\
\hline & Marta Lucía Ramírez (R) & $2,6 \%$ & $9,3 \%$ & $1,5 \%$ & $0,4 \%$ & $1,8 \%$ & & $0,8 \%$ & $0 \%$ \\
\hline & Clara López (L) & $1,4 \%$ & $11,4 \%$ & $1,9 \%$ & $0 \%$ & $3,8 \%$ & $1,5 \%$ & $0 \%$ & $0,3 \%$ \\
\hline & Enrique Peñalosa (P) & $1,6 \%$ & $12 \%$ & $0,4 \%$ & $0 \%$ & $0,4 \%$ & $5,2 \%$ & $0,4 \%$ & $0 \%$ \\
\hline \multirow{5}{*}{ El Tiempo } & Juan Manuel Santos (S) & $13,4 \%$ & $14,3 \%$ & $0,7 \%$ & $0 \%$ & $0 \%$ & $0,7 \%$ & $0,5 \%$ & $0,5 \%$ \\
\hline & Óscar Iván Zuluaga (Z) & $4,6 \%$ & $17,6 \%$ & $1,3 \%$ & $0,5 \%$ & $0 \%$ & $0,7 \%$ & $0 \%$ & $2 \%$ \\
\hline & Marta Lucía Ramírez (R) & $2,4 \%$ & $12,4 \%$ & $1,1 \%$ & $0 \%$ & $0 \%$ & $0,7 \%$ & $0 \%$ & $0,7 \%$ \\
\hline & Clara López (L) & $1,1 \%$ & $11,1 \%$ & $0,5 \%$ & $0 \%$ & $1,2 \%$ & $0,7 \%$ & $0,5 \%$ & $0 \%$ \\
\hline & Enrique Peñalosa (P) & $3,2 \%$ & $12,7 \%$ & $0 \%$ & $0 \%$ & $0 \%$ & $0,5 \%$ & $0 \%$ & $0 \%$ \\
\hline \multirow{5}{*}{$\begin{array}{l}\text { Caracol } \\
\text { Radio }\end{array}$} & Juan Manuel Santos (S) & $8,3 \%$ & $21,7 \%$ & $0 \%$ & $0 \%$ & $0 \%$ & $0 \%$ & $0 \%$ & $0 \%$ \\
\hline & Óscar Iván Zuluaga (Z) & $9,1 \%$ & $27,4 \%$ & $0 \%$ & $0 \%$ & $0 \%$ & $0 \%$ & $0 \%$ & $0 \%$ \\
\hline & Marta Lucía Ramírez (R) & $1,7 \%$ & $10,4 \%$ & $0 \%$ & $0 \%$ & $0 \%$ & $0 \%$ & $0 \%$ & $0 \%$ \\
\hline & Clara López (L) & $0 \%$ & $7,2 \%$ & $0 \%$ & $0 \%$ & $1,5 \%$ & $1,5 \%$ & $0 \%$ & $0 \%$ \\
\hline & Enrique Peñalosa (P) & $1,9 \%$ & $10,4 \%$ & $0 \%$ & $0 \%$ & $0 \%$ & $0 \%$ & $0 \%$ & $0 \%$ \\
\hline
\end{tabular}

Fuente: elaboración propia 
Este panorama permite entender la razón por la cual, durante los periodos de tiempo tomados para los tres medios, la mayoría de noticias para Santos y Zuluaga estuvieron dirigidas a un público sin especificación alguna, pero también fueron noticias de ataque de una campaña hacia la otra y viceversa. A diferencia de los tres candidatos restantes que, aunque presentan los porcentajes más altos dirigidos a un público en general, cuentan con noticias dirigidas hacia mujeres, campesinos, indígenas y afros con porcentajes más altos que las emitidas hacia alguno de sus homólogos. Es pertinente decir que Ramírez en todo momento se mostró como opositora a la campaña reeleccionista, y Peñalosa buscó crear oposición a Zuluaga, lo cual les genera que también tengan noticias emitidas hacia un dirigente político.

Estos resultados evidencian que los medios pueden limitar el conocimiento y la comprensión de una noticia por parte de la audiencia al no proponer información esencial y parcial sobre los candidatos (Rubido y Aparia, 1996), lo cual genera que esta persuasión implícita en la estructura de la noticia, acompañada de la ignorancia que tienen ciertas personas sobre el tema, se vuelva manipulación, pues como plantea Paniagua (2004), para que un elector pueda ejercer su derecho al voto con absoluta libertad debe, previamente, haber reflexionado sobre la información que recibió sin que esta haya sido limitada, pero como se hizo visible, las variables tomadas en cuenta mostraron la legitimación a través de las noticias que los candidatos Santos y Zuluaga obtuvieron, colaborando así en la aspiración de llegar a la segunda vuelta.

\section{Resultados segunda vuelta}

Tabla 6. Total de menciones de candidato por medio en segunda vuelta

\begin{tabular}{lcc}
\hline \multirow{2}{*}{$\begin{array}{c}\text { Candidato X medio } \\
\text { Caracol Televisión }\end{array}$} & Juan Manuel Santos (S) & Menciones \\
\cline { 2 - 3 } & Óscar Iván Zuluaga (Z) & $50,9 \%$ \\
\hline \multirow{2}{*}{ El Tiempo } & Juan Manuel Santos (S) & $51 \%$ \\
\hline \multirow{2}{*}{ Caracol Radio } & Óscar Iván Zuluaga (Z) & $48,9 \%$ \\
\hline
\end{tabular}

Fuente: elaboración propia

Para la segunda vuelta, se tomaron 34 noticias de Caracol Televisión en las que los candidatos fueron mencionados 53 veces, de las cuales el porcentaje más alto de menciones es ocupado por Zuluaga, sin desconocer que la diferencia con Santos es de 3,7 \%, lo cual permite ver que el medio televisivo, tanto en primera vuelta como en la segunda, fue equitativo entre las menciones emitidas de cada candidato. Por su parte, del periódico 
El Tiempo se tomaron 38 noticias que nombraban al par de candidatos 47 veces, de estas, Santos fue el candidato con el porcentaje más alto de menciones, pero al igual que con Caracol Televisión, la diferencia con las menciones del otro candidato es mínima (2,1%). Caso parecido sucedió con Caracol Radio de donde se tomaron 27 noticias y se nombró a los candidatos 31 veces, volviendo a ser Santos el candidato más mencionado, pero con una diferencia hacia su homólogo de 3,3 \%, demostrando que para el balotaje se buscó que las menciones de los candidatos tuvieran cierta equilibrio. En suma, este equilibrio en menciones por parte de los tres medios impidió que uno se destacara y lograra más legitimidad en el público, mientras que el otro iba despareciendo del área de juego, como se evidencio dentro de las elecciones para primera vuelta.

Tabla 7. Resultado de candidato por medio en segunda vuelta con las variables: lenguaje y tipo de anuncio

\begin{tabular}{|c|c|c|c|c|c|c|}
\hline \multirow{2}{*}{ Candidato X medio } & \multirow{2}{*}{ Variable } & \multicolumn{2}{|c|}{ Lenguaje } & \multicolumn{3}{|c|}{ Tipo de anuncio } \\
\hline & & Presente & Pasado & Positivo & Contraste & Negativo \\
\hline \multirow{2}{*}{ Caracol Televisión } & Juan Manuel Santos (S) & $47,2 \%$ & $0 \%$ & $34 \%$ & $7,5 \%$ & $3,8 \%$ \\
\hline & Óscar Iván Zuluaga (Z) & $47,2 \%$ & $3,7 \%$ & $26 \%$ & $13 \%$ & $9,4 \%$ \\
\hline \multirow{2}{*}{ El Tiempo } & Juan Manuel Santos (S) & $46,8 \%$ & $4,2 \%$ & $27,7 \%$ & $8,5 \%$ & $12,8 \%$ \\
\hline & Óscar Iván Zuluaga (Z) & $46,8 \%$ & $2,1 \%$ & $17 \%$ & $12,8 \%$ & $17 \%$ \\
\hline \multirow{2}{*}{ Caracol Radio } & Juan Manuel Santos (S) & $48,3 \%$ & $3,2 \%$ & $25,8 \%$ & $16,1 \%$ & $6,5 \%$ \\
\hline & Óscar Iván Zuluaga (Z) & $48,3 \%$ & $0 \%$ & $19,4 \%$ & $9,7 \%$ & $12,9 \%$ \\
\hline
\end{tabular}

Fuente: elaboración propia

Los porcentajes más altos en los tres medios de comunicación para ambos candidatos, utilizaron lenguaje en presente, sin desconocer que, en Caracol Televisión, Zuluaga presentó noticias con lenguaje en pasado mientras que las de Santos fueron todas en presente. Por parte del periódico El Tiempo y Caracol Radio, Santos es quien tiene mayor porcentaje de noticias en pasado frente a las noticias en pasado de Zuluaga. No obstante, aunque las noticias en pasado se definen con tendencia a ser negativas, en la variable de tipo de anuncio, es Zuluaga quien presenta los porcentajes más altos de noticias negativas en los tres medios de comunicación, frente a los resultados de su homólogo.

Como la apatía y manipulación a causa de la saturación de información y el frecuente enfrentamiento entre candidatos produjo un debilitamiento en el compromiso cívico de las personas (Pereira, 2007), Santos y Zuluaga buscaron esta vez cambiar las campañas negras de desprestigio entre ambos y dedicarse más a sus propuestas, no obstante, Santos se enfocó para el balotaje en el tema de la paz que fue tomado como coyuntura política, mientras que Zuluaga tuvo más ataques directos hacia su opositor con un enfoque de sus noticias de cotidianidad. 
Tabla 8. Resultado de candidato por medio en segunda vuelta con la variable: tema

\begin{tabular}{|c|c|c|c|c|}
\hline \multirow{2}{*}{\multicolumn{2}{|c|}{ Candidato X medio $\quad$ Variable }} & \multicolumn{3}{|c|}{ Tema } \\
\hline & & $\mathrm{CP}$ & $\mathrm{C}$ & CSFC \\
\hline \multirow{2}{*}{ Caracol TV } & Juan Manuel Santos (S) & $26,4 \%$ & $18,9 \%$ & $1,9 \%$ \\
\hline & Óscar Iván Zuluaga (Z) & $18,9 \%$ & $30,2 \%$ & $1,9 \%$ \\
\hline \multirow{2}{*}{ El Tiempo } & Juan Manuel Santos (S) & $27,2 \%$ & $23,4 \%$ & $0 \%$ \\
\hline & Óscar Iván Zuluaga (Z) & $14,8 \%$ & $31,9 \%$ & $2,1 \%$ \\
\hline \multirow{2}{*}{ Caracol Radio } & Juan Manuel Santos (S) & $16,1 \%$ & $35,3 \%$ & $0 \%$ \\
\hline & Óscar Iván Zuluaga (Z) & $9,7 \%$ & $35,5 \%$ & $3,2 \%$ \\
\hline
\end{tabular}

Fuente: elaboración propia

Tabla 9. Resultado de candidato por medio en segunda vuelta con la variable: a quién va dirigido

\begin{tabular}{|c|c|c|c|c|c|c|c|c|c|}
\hline \multirow{2}{*}{ Candidato X medic } & \multirow[t]{2}{*}{ Variable } & \multicolumn{8}{|c|}{ A quién va dirigido } \\
\hline & & DP & $P G$ & EU & FFMM & M & CIA & VV & $\mathrm{PF}$ \\
\hline \multirow[b]{2}{*}{ Caracol TV } & Juan Manuel Santos (S) & $5,60 \%$ & $34 \%$ & $3,70 \%$ & $1,9 \%$ & $0 \%$ & $3,7 \%$ & $0 \%$ & $0 \%$ \\
\hline & Óscar Iván Zuluaga (Z) & $9,4 \%$ & 43,3 & $1,9 \%$ & $1,9 \%$ & $0 \%$ & $3,7 \%$ & $0 \%$ & $0 \%$ \\
\hline \multirow{2}{*}{ El Tiempo } & Juan 1 & $\begin{array}{c}12,8 \\
\%\end{array}$ & 42,6 & $0 \%$ & $0 \%$ & $0 \%$ & $0 \%$ & $0 \%$ & $0 \%$ \\
\hline & Óscar Iván Zuluaga (Z) & $6,3 \%$ & 426 & $0 \%$ & $0 \%$ & $0 \%$ & $0 \%$ & $0 \%$ & $0 \%$ \\
\hline \multirow{2}{*}{ Caracol Radio } & Juan Manuel Santos (S) & $6,5 \%$ & $\begin{array}{c}38,7 \\
\%\end{array}$ & $0 \%$ & $\%$ & $0 \%$ & $6,5 \%$ & $0 \%$ & $\begin{array}{c}3,2 \\
\%\end{array}$ \\
\hline & Óscar Iván Zuluaga (Z) & $\begin{array}{c}12,9 \\
\%\end{array}$ & $29 \%$ & $0 \%$ & $0 \%$ & $0 \%$ & $6,5 \%$ & $0 \%$ & $0 \%$ \\
\hline
\end{tabular}

Fuente: elaboración propia

Por último, aunque los dos candidatos vuelven a enfocar sus noticias en la segunda vuelta hacia un público en general, también tienen noticias dirigidas hacia su contrincante político, destacándose Zuluaga por ser el candidato con más ataques hacia su homólogo en el medio televisivo y radial, mientras que Santos hace lo propio en el medio escrito. Cabe decir que el resultado para los dos candidatos es mayor al de sus menciones ya que obtuvieron varias noticias que estuvieron dirigidas a ambos grupos a la vez.

Según datos obtenidos de la Registraduría Nacional, Santos fue el ganador con 7.836.987 votos frente a los 6.905.001 votos que tuvo Zuluaga para el balotaje, en cifras porcentuales es un 50,75\% para Santos frente a un 46,69 \% de Zuluaga, lo que indica que en segunda vuelta los dos candidatos recibieron alrededor del doble de votos obtenidos en la primera, así, al relacionar estos resultados con las variables seleccionadas, permite identificar que el hecho de enfocar sus discursos más en sus propuestas de campaña que en los ataques directos de desprestigio hacia su homólogo, es uno de los factores relevantes en el aumento de la participación de los votantes quienes en primera vuelta se vieron envueltos por parte de los medios de comunicación en una exposición de noticias enfocadas en dos campañas que se destacaron por tener porcentajes altos de anuncios negativos dirigidos hacia la campaña de oposición y limitando la votación de la población 
más hacia el clima de opinión que a las propuestas políticas, todo ello causó un abstencionismo de $60 \%$ que se redujo a $52 \%$ en segunda vuelta.

\section{Conclusiones}

Referente a los medios de comunicación, es pertinente reconocer que su influencia no se ejerce de manera directa, lo cual se evidenció en primera vuelta cuando Santos con mayoría de noticias positivas y con lenguaje presente es superado por Zuluaga en los resultados de primera vuelta, sin embargo, tomado desde Van Dijk (1994), se puede decir que el poder de los medios es simbólico y persuasivo, pero el control lo hacen de manera indirecta cuando ejercen un dominio en las intenciones de proyectos, creencias y opiniones, a través de las categorías dentro de los discursos para que las elites se legitimen y tengan el control sobre el espectador, tal como se vio en todo este proceso electoral pues "cuando el periódico repite más o menos lo que dicen los políticos o la clase dirigente se crea un círculo muy difícil de romper" (Van Dijk, 1994, p. 43) pero esto no solo se hizo evidente con el medio escrito, sino también con el televisivo y el radial, donde muy poco espacio se le proporcionó al análisis y terminaron presentando noticias netamente informativas que llevaron consigo todo tipo de categorías y simbolismos, generando un estatus a cada uno de los candidatos.

Teniendo en cuenta cómo se desarrollaron las noticias para todos los candidatos, existe un deterioro en la democracia electoral, concepto tomado desde Larry Diamond (2003), puesto que para este autor, una democracia electoral debe asegurar que los cargos públicos de poder político deben gestionarse mediante elecciones periódicas, libres, limpias, justas y competitivas, sin embargo, limitándose a la estructuración de las noticias emitidas por los tres medios analizados y sin desconocer que el autor plantea esta definición desde parámetros sociales, culturales y políticos haciendo referencia a la función institucional, es posible analizar cómo las elecciones dentro de los medios de comunicación no son lo suficientemente justas y libres ya que la información que le está llegando a la población ha sido previamente seleccionada y categorizada lo que impide una oportunidad justa entre los candidatos y la posibilidad de un análisis libre y críitico por parte de la población.

Si las libertades de expresión e información son indispensables en una sociedad democrática (...) el Estado debe velar porque los ciudadanos puedan tener los elementos necesarios para emitir su voto de manera racional tras haber recibido de forma apropiada la información referida a su entorno social y político (Holgado, 2003, p. 47).

Ahora bien, teniendo en cuenta los resultados de las elecciones tanto de primera como de segunda vuelta, cabe considerar si es pertinente tomar como voluntad de la población que solo un pequeño número de personas sea el que elija a un gobernante, o más bien, si los altos porcentajes de abstención, están queriendo decir algo y el régimen democrático electoral no está respondiendo adecuadamente a las situaciones electorales que se presentan en el país. 


\section{Referencias}

Aceves, F. (1993). La influencia de los medios en los procesos electorales: una panoramica desde la perspectiva de la sociologia empirica. Comunicación y sociedad, (18), 223-255.

Berrocal, S. (2008). El discurso de la politica espectáculo en televisión. Un análisis de "tengo una pregunta para usted": investigar la comunicación Valladolid: Universidad Valladolid.

Diamond, L. (2003). ¿Puede el mundo entero ser democrático? Revista Española de Ciencia Política, (9), 9-38.

García, V., D’Adamo, O. y Slavinsky, J. (2005). Comunicación política y campañas electorales, análisis de una herramienta comunicacional: estrategias en elecciones presidenciales. Barcelona: Gedisa.

Gauthier, G. (1998). Comunicación y política. Barcelona: Gedisa.

González, J. (1989). El espectáculo informativo o la amenaza de lo real. Madrid: Akal Comunicación.

Holgado, M. (2003). El papel de los medios de comunicación en la campaña electoral. Ámbitos, Revista Internacional de Comunicación, 10, 32-55.

Lazarsfeld, P., Berelson, B. y Gaudet H. (1949). The people's choice: how the voters makes up his maind in a presidential campaign. Nueva York: Columbia University Press.

McCombs, M. y Shaw, D. (1977). The emergence of american political issues: the agenda setting function of the press. Eagan: West Group.

Neumann, N. (1995). La espiral del silencio. Opinión pública: nuestra pile social. Barcelona: Paidós.

Paniagua, F. (2004). La nueva comunicacion electoral en España. Revista Latina de Comunicacion Social. $7(58), 1-14$.

Pereira, J. (2007). Elecciones, campañas y medios de comunicación. Granada: Universidad de Granada.

Rodríguez, X. (2011). Resumen de the effects of mass comunication. Razón y Palabra. (75), 1-17. Recuperado de http://www.razonypalabra.org.mx/N/N75/monotematico_75/30_Rodriguez_M75.pdf

Semana. (2014). El balance del primer Santos. Recuperado de http://www.semana.com/nacion/articulo/ el-balance-del-primer-santos/398507-3

Serrano, M. (2014). Elecciones presidenciales en Colombia 2014. Instituto Español de Estudios Estratégicos, (68). Recuperado de http://www.ieee.es/en/Galerias/fichero/docs_opinion/2014/ DIEEEO68-2014_EleccionesColombia_MASM.pdf

Tamayo, C. (2007). Mutaciones contemporáneas: proceso electoral y medios de comunicación en Colombia 2006. En T. Mejía, E. Torrico, K. Herrera, E. Gerbet, A. Araya y N. Guzmán (Eds.), Se nos rompió el amor (elecciones y medios de comunicación -América Latina 2006) (pp.117-150). Bogotá: Centro de Competencia en Comunicación para América Latina.

Van Dijk, T. (1994). Discurso, poder y cognición social. Recuperado de https://www.textosenlinea.com. ar/academicos/Discurso\%20poder\%20y\%20cognici\%C3\%B3n\%20social.pdf

Vega, M. (2010). La centralidad de la televisión en el terreno de la comunicación política. Barcelona: Universidad Autónoma de Barcelona. 